\title{
Microswimmers - From Single Particle Motion to Collective Behavior
}

\author{
Gerhard Gompper ${ }^{1, a}$, Clemens Bechinger ${ }^{2}$, Stephan Herminghaus ${ }^{3}$, \\ Rolf Isele-Holder ${ }^{1}$, U. Benjamin Kaupp ${ }^{4}$, Hartmut Löwen ${ }^{5}$, Holger Stark ${ }^{6}$, \\ and Roland G. Winkler ${ }^{1}$ \\ 1 Theoretical Soft Matter and Biophysics, Institute of Complex Systems \& Institute for \\ Advanced Simulation, Forschungszentrum Jülich, 52425 Jülich, Germany \\ 2 Physikalisches Institut, Universität Stuttgart, 70550 Stuttgart, Germany \\ 3 Max-Planck-Institut für Dynamik und Selbst-Organisation, 37077 Göttingen, Germany \\ 4 Forschungszentrum caesar, 53175 Bonn, Germany \\ 5 Institut für Theoretische Physik, Heinrich-Heine-Universität, 40225 Düsseldorf, Germany \\ ${ }^{6}$ Institut für Theoretische Physik, Technische Universität Berlin, 10623, Berlin, Germany
}

Received 29 March 2016 / Received in final form 29 March 2016

Published online 10 November 2016

\begin{abstract}
Locomotion of autonomous microswimmers is a fascinating field at the cutting edge of science. It combines the biophysics of selfpropulsion via motor proteins, artificial propulsion mechanisms, swimming strategies at low Reynolds numbers, the hydrodynamic interaction of swimmers, and the collective motion and synchronisation of large numbers of agents. The articles of this Special Issue are based on the lecture notes of an international summer school, which was organized by the DFG Priority Programme 1726 "Microswimmers - From Single Particle Motion to Collective Behaviour" in the fall of 2015. The minireviews provide a broad overview of the field, covering both elementary and advanced material, as well as selected areas from current research.
\end{abstract}

\section{Introduction}

Locomotion and transport of microorganisms in fluids is an essential aspect of life. Search for food, orientation toward light, spreading of off-spring, and the formation of colonies are only possible due to locomotion. Microorganisms, such as bacteria, algae, and sperm cells exploit flagella for their propulsion. Swimming at this microscale occurs at low Reynolds numbers, where fluid friction and viscosity dominates over inertia. This requires swimming strategies different from those of the macroscopic world. Evolution achieved propulsion mechanisms, which overcome and even exploit drag. Two prominent swimming mechanisms are rotating helical flagella, exploited by many bacteria, and snake-like or whip-like motion of eukaryotic flagella, utilized by sperm and algae.

\footnotetext{
a e-mail: g.gompper@fz-juelich.de
} 
The understanding of these propulsion mechanisms opens an avenue for control of biological systems and the design of artificial nanomachines, with a major impact on various fields ranging from life science and material science to environmental science. For artificial microswimmers, alternative concepts to convert chemical energy or heat into directed motion can be employed, which are potentially more efficient.

The dynamics of microswimmers comprises many facets, which are all required to achieve locomotion. On the level of an individual swimmer, the propulsion mechanism needs to be unravelled. Thereby the question of the energy supplied for persistent motion has to be addressed. The response to external stimuli by chemical signals, light or gravitational fields, or flow is another important issue. A major challenge is the understanding and control of the emergent collective behavior of microswimmers. Here, the formation of large-scale patterns as networks and swarms in microswimmer assemblies needs to be resolved.

We are at a very interesting moment in the historical development of microswimmer research. While the motion of single sperm and bacteria has been studied both experimentally and theoretically for about half a century, advanced experimental and simulation techniques open an avenue for a deeper understanding of the underlying physical and bio-chemical processes. Furthermore, nanotechnology, and softmatter chemistry and physics now provide the tools to design and construct artificial microswimmers reproducibly and with high precision. Novel simulation approaches allow for the investigation of large ensembles of microswimmers together with the fluid-mediated interactions. Finally new statistical theories have been developed to tackle non-equilibrium many body problems. Microswimmer research has become an interdisciplinary endeavor of biology, biophysics, theoretical and experimental soft matter physics, and simulation sciences.

Consequently, major research goals nowadays are:

- understanding biological microswimmers;

- designing and understanding artificial microswimmers;

- understanding cooperative behavior and "swarming" of ensembles of many microswimmers.

To address these challenges requires a synergetical combination of experimental, theoretical, and simulation expertise. There are several related systems, in which similar mechanisms are at play and similar types of structures appear. On the mesoscale, these are, for example, mixtures of biological filaments and motor proteins, and vibrated granular systems.

\section{Special Issue}

The minireviews presented in this Special Issue are based on the lecture notes of an international summer school, which was organized by the DFG Priority Programme 1726 "Microswimmers - From Single Particle Motion to Collective Behaviour" in the fall of 2015. The articles should therefore be well suited for students and young researchers who want to gain an overview of the field, but also for experts who are interested in a more detailed look into some of the new research directions in a fascinating and very quickly developing research field.

In this way, we hope to widen horizons to encompass the truly interdisciplinary field of microswimmers and self-propelled particles. This includes in particular introducing biologists and chemists to physical experimental methods and theoretical modelling, and introducing physicists to a large variety of fascinating chemical and biological phenomena. 


\subsection{Theory and concepts}

Microswimmers are immersed in a solvent and show collective phenomena when present in a large group. Generic aspects, specifically the foundations of hydrodynamic interactions at low Reynolds numbers and the general principles of non-equilibrium statistical mechanics and collective motion, are covered in a few minireviews [1-3].

\subsection{Biological microswimmers}

Evolution has generated a large diversity of biological microswimmers, each using propulsion mechanisms and navigation strategies tailored to their natural environment and function. Understanding the complex swimming strategies of biological swimmers potentially enables controlling the behaviour of these swimmers in technical applications. Moreover, propulsion and synchronization mechanisms from natural swimmers can be transferred and even optimized in the design of artificial swimmers. Various minireviews provide an overview of different classes of natural swimmers [4-7].

\subsection{Artificial microswimmers}

Self-propulsion requires the conversion from chemical to kinetic energy. First technical realizations of self-propelled objects have been developed for different driving mechanisms and conversion strategies. The fundamental driving mechanisms as well as state-of-the-art technologies of artificial microswimmers are covered in several minireviews [8-13].

\subsection{Collective behaviour}

Microswimmers cannot accomplish their tasks without their ability to spontaneously organize coordinated and collective motion when present in large groups. This collective behavior has generic aspects, like the general tendency of microswimmers for cluster formation and motility-induced phase separation, but also many specific aspects for different types of microswimmers. Therefore, various model and biological systems have to be studied in detail. In particular, the impact of swimmer shape, wall effects, effects of the surrounding media, and effects caused by external gravitational or flow fields are presented in several minireviews [14-20].

\section{Outlook}

There is a large variety of future challenges which need to be tackled in the field of microswimmers and self-propelled particles. For example, artificial microswimmers are nowadays often restricted to special reactive media for propulsion; thus, new systems have to be developed which are more universally deployable in applications. Artificial microswimmers also have to become "smarter", so that they can react and adapt to their environment. Here, a combination of artifical and biological microswimmers or swimming mechanisms in hybdrid systems may be a promising option. Microswimmers are small - therefore they can also only carry a small payload or perform a small workload. Thus, swarms of self-propelled particles are necessary to perform a task, and this swarming has to be facilitated and controlled. A step even further 
are smart materials composed of microswimmers, and we are just at the beginning of understanding the physical properties of such systems. Finally, there is a wealth of possible applications in biology and medicine, the potential of which needs to be expored and exploited, for example in drug delivery and cancer treatment.

This work was supported by the DFG priority program SPP 1726 on "Microswimmers from Single Particle Motion to Collective Behaviour".

\section{References}

1. D. Marenduzzo, Eur. Phys. J. Special Topics 225, 2065 (2016)

2. R.G. Winkler, Eur. Phys. J. Special Topics 225, 2079 (2016)

3. F. Ginelli, Eur. Phys. J. Special Topics 225, 2099 (2016)

4. U.B. Kaupp, L. Alvarez, Eur. Phys. J. Special Topics 225, 2119 (2016)

5. R. Jeanneret, M. Contino, M. Polin, Eur. Phys. J. Special Topics 225, 2141 (2016)

6. T. Krüger, M. Engstler, Eur. Phys. J. Special Topics 225, 2157 (2016)

7. S. Klumpp, D. Faivre, Eur. Phys. J. Special Topics 225, 2173 (2016)

8. M. Popescu, W. Uspal, S. Dietrich, Eur. Phys. J. Special Topics 225, 2189 (2016)

9. K. Kroy, D. Chakraborty, F. Cichos, Eur. Phys. J. Special Topics 225, 2207 (2016)

10. R. Seemann, J.-B. Fleury, C.C. Maass, Eur. Phys. J. Special Topics 225, 2227 (2016)

11. M. Alarcón-Correa, D. Walker, T. Qiu, P. Fischer, Eur. Phys. J. Special Topics 225, $2241(2016)$

12. J. Parmar, D. Vilela, S. Sanchez, Eur. Phys. J. Special Topics 225, 2255 (2016)

13. H.-H. Boltz, J. Kierfeld, Eur. Phys. J. Special Topics 225, 2269 (2016)

14. T. Speck, Eur. Phys. J. Special Topics 225, 2287 (2016)

15. F. Peruani, Eur. Phys. J. Special Topics 225, 3001 (2016)

16. H. Löwen, Eur. Phys. J. Special Topics 225, 3019 (2016)

17. J. Elgeti, G. Gompper, Eur. Phys. J. Special Topics 225, 3033 (2016)

18. B. Friedrich, Eur. Phys. J. Special Topics 225, 3053 (2016)

19. H. Stark, Eur. Phys. J. Special Topics 225, 3069 (2016)

20. E. Clement, A. Linder, C. Douarche, H. Auradou, Eur. Phys. J. Special Topics 225, 3089 (2016) 